\title{
Combining radiation therapy and immunotherapy for lung cancers: a narrative review
}

\author{
Chirag Modi ${ }^{1}$, Lyudmyla Berim ${ }^{2}$, Lauren Isserow ${ }^{1}$, Jyoti Malhotra ${ }^{2}$, Malini Patel ${ }^{2}$, John Langenfeld $^{3}$, \\ Joseph Aisner ${ }^{2}$, Doaa Almeldin', Salma K. Jabbour ${ }^{1}$
}

${ }^{1}$ Department of Radiation Oncology, ${ }^{2}$ Division of Medical Oncology, ${ }^{3}$ Division of Surgical Oncology, Rutgers Cancer Institute of New Jersey, Rutgers Robert Wood Johnson Medical School, Rutgers University, New Brunswick, NJ, USA

Contributions: (I) Conception and design: SK Jabbour, C Modi; (II) Administrative support: SK Jabbour; (III) Provision of study materials or patients: None; (IV) Collection and assembly of data: C Modi, L Berim, D Almeldin, L Isserow; (V) Data analysis and interpretation: All authors; (VI) Manuscript writing: All authors; (VII) Final approval of manuscript: All authors.

Correspondence to: Salma K. Jabbour. Department of Radiation Oncology, Rutgers Cancer Institute of New Jersey, Robert Wood Johnson Medical School, Rutgers University, New Brunswick, NJ, USA. Email: jabbousk@cinj.rutgers.edu.

\begin{abstract}
Lung cancer remains the leading cause of cancer morbidity and mortality worldwide among both men and women. While surgical resection remains the standard of care for early stage NSCLC, chemoradiation has been a mainstay of treatment for locally advanced non-small-cell lung cancer (LA-NSCLC) patients for decades. Consolidation immunotherapy has improved survival in this subset of patients after conventional chemoradiation, and has emerged as the new standard. The synergy between immunotherapy and radiation, as well as ongoing research on the effects of radiation on the immune system, allows for the exploration of new avenues in the treatment of LA-NSCLC. In addition to the use of durvalumab as consolidative systemic therapy after concurrent chemoradiotherapy for Stage III NSCLC, other combination regimens have been shown to be effective in various disease stages in preclinical and clinical studies. These regimens include CTLA-4 and PD/PDL-1 checkpoint inhibitors combined with radiation treatment. While these combined regimens have demonstrated efficacy, they are not without toxicity, and require additional evaluation when combined with radiation. In this review, we have summarized the immunostimulatory and immunosuppressive effects of radiation therapy. We also evaluate the current evidence and ongoing research supporting the combination of radiotherapy and immunotherapy across early to LA-NSCLC.
\end{abstract}

Keywords: Immunotherapy; radiotherapy; stereotactic body radiation therapy (SBRT); non-small cell lung cancer; chemoradiation

Received: 27 April 2020; Accepted: 07 August 2020; Published: 10 January 2021.

doi: $10.21037 /$ shc-20-66

View this article at: http://dx.doi.org/10.21037/shc-20-66

\section{Background}

Lung cancer is the most common cancer and a leading cause of cancer related death in the world. In 2018, there were approximately 2.1 million new cases of lung cancer diagnosed worldwide, and a 1.8 million lung cancer related deaths (1). Surgical resection remains the standard of care for treatment of stage I non-small cell lung cancer (NSCLC), with reported long-term disease-free survival (DFS) rates of $80-90 \%(2-4)$. In patients with early stage
NSCLC who are deemed not to be candidates for surgery or those who refuse surgical intervention, stereotactic body radiation therapy (SBRT) has been demonstrated to be potentially comparable, with local control rates approaching $90 \%$ in some series $(5,6)$. By contrast, definitive chemoradiotherapy followed by consolidative therapy with durvalumab, an anti-programmed death ligand 1 (PD-L1) antibody, has become the new standard of care for locally advanced unresectable NSCLC $(7,8)$. SBRT has allowed for high doses of highly conformal radiation delivered in 
a limited number of fractions to a tumor, facilitating the precision of therapy while minimizing radiation dose to adjacent organs at risk.

Radiation therapy (RT) has been shown to have a number of immunostimulatory effects, including priming the immune system, recruiting immune cells, and altering the immunosuppressive effects of the tumor microenvironment (9). In this review, we will summarize evidence and ongoing research on combining immunotherapy with conventional radiation or SBRT across early stage and locally advanced non-small cell lung cancer.

\section{Methods}

We performed a literature search using PubMed on radiation therapy and immunotherapy and also searched ClinicalTrials.Gov for ongoing and completed trials on the subject. Additionally, we evaluated research from major oncology conferences including ASTRO, ASCO, and ESMO to further compile a comprehensive list of studies. Relevant articles and studies were reviewed by the authors and their results were summarized.

We present the following article in accordance with the Narrative Review reporting checklist (available at http:// dx.doi.org/10.21037/shc-20-66).

\section{Effects of radiation on cancer immunity}

Radiation therapy is known to induce cellular demise by inflicting double strand DNA damage mediated through a variety of mechanisms, including apoptosis, mitotic catastrophe, and radiation-induced senescence (10). Cell death triggers a tightly knitted series of immune events, both immunostimulatory and immunosuppressive. There are key major pathways mediating an RT induced immune response such as dendritic cell activation via tumor antigen presentation, increase in tumor-infiltrating lymphocytes, and modulation of extracellular signaling $(7,8)$. New treatment regimens are being developed on the basis of these mechanisms.

\section{Activation of dendritic cells}

At the molecular level, ionizing radiation induces irreparable DNA damage within tumor cells, which in a dose responsive manner results in expansion of the intracellular peptide pool, expression of calreticulin, upregulation of the surface MHC class 1 molecules, leading to phagocytosis by dendritic cells (DC), DC activation, enhanced tumor antigen cross-presentation and promotion of inflammatory cytokines secretion $(11,12)$. It has been shown that the process of uptake and cross-presentation of tumor-derived antigens by DC to T-cells, the RTmediated tumor regression and adaptive immune response to RT is mediated by type I interferon (IFN-I) (13). The endoplasmic reticulum-associated stimulator of interferon genes (STING) protein has been demonstrated to be a mediator for induction of IFN-I by intracellular exogenous DNA (14,15). Burnette et al. demonstrated the ability of radiation to increase the expression of IFN-I (16). In mice without an IFN-B receptor, the ability of RT to reduce tumor growth was diminished. Therefore, DCs are central to the downstream effects of RT via IFN-1 and IFN-B.

\section{Upregulation of $C D 8+T$ cells}

After recruitment and activation by RT-induced tissue damage, DCs migrate to the tumor-draining lymph nodes and present tumor-associated antigens (TAA) to CD8+ T cells. This presentation results in enhanced expansion and activation of the cytotoxic $T$ cell pool comprised of tumor-specific effector CD8+ T cells (11). Radiation induces production of chemokines CXCL10 and CXCL16 by tumor cells and vascular cell adhesion molecules, VCAM-1 and ICAM, by endothelial cells, thereby facilitating trafficking, homing and adherence of the tumor-specific effector $T$ cells to the irradiated tissue (16,17-22), as well as cancer lesions outside of the RT field, also contributing to the abscopal effect (23).

\section{Induction of apoptosis}

Another important mechanism of interaction between radiation and the immune system is the modulation of extracellular signaling at the time of cell death through the First Apoptosis Signal (FAS) pathway activation. FAS is a cell-surface molecule that induces programmed cell death through combining with FAS-Ligand (FAS-L). Subsequent activation of caspases 3, 6, and 7 leads to apoptotic cell death. Chakraborty et al. demonstrated upregulation of the FAS pathway in tumor cells by sublethal irradiation, resulting in improved lytic susceptibility to CD8+ cells and promotion of more effective antitumor responses in vivo (24,25). By contrast, several studies have shown that RT upregulates FAS-L on endothelial cells to preferential apoptosis in $\mathrm{T}$ effector cells while sparing $\mathrm{T}$ regulator cells, thereby creating a pro-tumoral and immunosuppressive 
environment $(26,27)$. Thus, the depletion of FAS-L expression on tumor endothelial cells can potentially lead to an increase in CD8+ T cells entering the tumor microenvironment (26). Other radiation-induced death receptors have also been identified, including tumor necrosis factor-related apoptosis-inducing ligand (TRAIL), as well as CD-80 and the natural killer group 2D (NKG2D) ligands, activation of which may lead to clearance of cancer cells by NK cells (28-30).

\section{Immunosuppressive effects}

Ionizing radiation also may have immunosuppressive properties. Local immune response to RT may be dampened as lymphocytes are sensitive to radiation doses and are cleared rapidly from the radiation field (31). Treatmentrelated lymphopenia is commonly seen in patients receiving RT (32). Preclinical studies have shown that cytotoxic $\mathrm{T}$-cells are more radiosensitive than regulatory $\mathrm{T}$-cells which can lead to a disproportionally higher number of regulatory T-cells and suppresses immune response. Novel strategies targeting regulatory $\mathrm{T}$ cells (Treg), tumorassociated macrophages, and cancer-associated fibroblasts in combination with radiation treatment and immunotherapy are being investigated $(17,21,22,33)$.

\section{Radiation doses and timing}

Dose per fraction and fractionation schemes can also affect the immune response induced by radiation. Shaue $e t$ al. showed a dose dependent tumor control and increase in number of tumor-reactive $T$ cells in a spleen of a murine melanoma model. However, at high RT doses beyond 7.5 Gy per fraction, researchers observed an increased representation of Tregs, which abrogated further immune response (34). High RT doses of greater than 18-20 Gy per fraction induce DNA exonuclease Trex1, which degrades cytosolic DNA and prevents activation of STING pathway, therefore suppressing radiotherapy-induced tumor immunogenicity (35). Preclinical data has shown that SBRT doses in the range of 12-18 Gy can lead to an upregulation of exonuclease in Trex1, but fail to do so at lower doses (35). Lugade et al. showed that 15 Gy single dose irradiation resulted in greater numbers of host immune cell infiltrate on Day 14 than a 5 Gy $\times 3$ fractionation in a B16 melanoma model (17). Despite these preclinical models, the optimal fractionation schemes in combination with immunotherapy require further clinical evaluation.

\section{Evidence for immunoradiotherapy in NSCLC}

One important hallmark of cancer is the ability of neoplastic cells to evade immunological destruction by lymphocytes, macrophages, and natural killer cells (36). Over the past decade various approaches to increase cancer immunogenicity have been explored. Radiotherapy can increase tumor immunogenicity by the upregulation of antigen expression and recognition by immune cells, overcoming an immunosuppressive tumor microenvironment via enhanced production of immunostimulatory cytokines, and engaging antigenpresenting and immune effector cells within the tumor microenvironment (36). Antitumor immunity induced by local RT as a single modality is most often insufficient to achieve complete and long-lasting tumor clearance. Synergy between radiation therapy and immunotherapy has been therefore explored in clinical trials with various malignancies, including lung cancer. Immune checkpoint blockade (ICB) has recently changed the paradigm of our therapeutic approach to the management of multiple neoplastic diseases. Increased recognition of favorable effects of RT immunogenicity stimulated interest and development in a spectrum of clinical trials studying synergism between the two therapeutic strategies.

\section{CTLA-4 inbibitors in combination with radiation therapy}

Cytotoxic T-lymphocyte-associated protein 4 (CTLA4 , CD152), is a protein lymphocyte surface receptor that is a CD28 homolog with high affinity for B7-1 and B72. In contrast to the interaction of CD28 with $\mathrm{B} 7-1 / 2$, which transmits a stimulatory signal for $\mathrm{T}$ cell proliferation and activation, the binding of CTLA- 4 to $\mathrm{B} 7-1 / 2$ results in inhibition of the early $\mathrm{T}$ cell activation. CTLA-4 is a key negative regulator of $\mathrm{T}$ cell activation, acting as an immune checkpoint. Following engagement with $\mathrm{T}$ cell receptor, CTLA-4 can translocate to the cell surface, where it outcompetes CD28 for binding to critical costimulatory molecules (CD80, CD86) and mediates inhibitory signaling into the $\mathrm{T}$ cell, resulting in arrest of both proliferation and activation (37). CTLA-4 blockade potentiates T cell proliferation and activation and leads to production of immuno-stimulatory molecules including IFN-gamma, IL2 , and TNF-alpha $(38,39)$. Ji et al. showed an increase in tumor-infiltrating lymphocytes and INF-gamma levels with anti-CTLA-4 blockade (40).

CTLA-4 blocking antibody has been combined with 
radiation therapy to study a potential synergistic response in pre-clinical setting (41-43). Dewan et al. tested various dose fractionation regimens to determine the synergistic antitumor effect between radiation and 9H10 monoclonal antibody against CTLA-4 (42). Specifically, breast and colon carcinoma mouse models were treated with three distinct regimens of RT $(20 \mathrm{~Gy} \times 1,8 \mathrm{~Gy} \times 3$, or $6 \mathrm{~Gy} \times 5)$ in combination with a 9H10 CTLA-4 antibody versus $9 \mathrm{H10}$ CTLA-4 antibody alone. While the CTLA-4 antibody alone had no detectable effect on tumor size, the combination of the CTLA-4 antibody with all of the three fractionated radiotherapy regimens led to significant growth inhibition of the tumor $(\mathrm{P}<0.001)$. Additionally, a significant decrease in size of the tumor outside the radiation field, or the abscopal effect, was observed in mice treated with the combination of CTLA-4 antibody and fractionated radiotherapy. The frequency of CD8+ T cells showing tumor-specific IFNgamma production was found to be proportional to the inhibition of the distant secondary tumor (42).

The aforementioned preclinical work led to multiple clinical trials assessing the feasibility of combining CTLA-4 blockade and RT. A phase I study completed by Tang et al. combined ipilimumab with SBRT in different fractionation schemes (41). In the study, 31 patients with metastatic solid tumors to the lung and liver received $3 \mathrm{mg} / \mathrm{kg}$ of ipilimumab every 3 weeks for 4 doses with 50 Gy in 4 fractions or 60 Gy in 10 fractions concurrently or sequentially. Combination therapy was found to be safe and effective, demonstrating $10 \%$ out-of-field immune-related partial response and $23 \%$ clinical benefit. Additionally, increased peripheral T-cell activation was observed in patients deriving clinical benefit.

Formenti et al. studied biology of abscopal response in thirty-nine patients with refractory metastatic NSCLC treated with RT to a single metastatic lesion concurrently with ipilimumab (44). Objective systemic responses were observed in $18 \%$ of patients and an additional $31 \%$ of patients had disease control. Increased serum interferon- $\beta$ after RT, rapid in vivo expansion and persistence of a large number of tumor-specific $\mathrm{T}$ cell clones in peripheral blood were shown to be the strongest predictors of response, further supporting hypothesis of RT-induced immunogenicity and the abscopal phenomenon (44).

\section{PD-1 or PDL-1 inbibitors in combination with $R T$}

Programmed death receptor-1 protein (PD-1) is a cell surface receptor, a member of the CD28 superfamily, that delivers inhibitory signal to $\mathrm{T}$ cell activation through binding to soluble ligands PD-L1 and PD-L2. In contrast to CTLA-4, which is only expressed on $\mathrm{T}$ cells thereby regulating early immune response primarily in lymph nodes, PD-1 is expressed by $\mathrm{T}$ cells, B cells, macrophages, and some tumor cells. PD-1 regulates $\mathrm{T}$ cell effector responses in the peripheral tissues, whereby CTLA-4 controls T cells activation. Similarly to CTLA-4, PD-1 signaling promotes apoptosis in antigen-specific T-cells, interferes with $\mathrm{T}$ cell proliferation and cytokine signaling, and reduces apoptosis of T regulatory cells (45). One of the signs of loss of effector function and $\mathrm{T}$ cell exhaustion is increased expression of PD-1 (46). In addition to CTLA-4 inhibitors, PD-1 and PDL-1 inhibitors have also been shown to have a synergistic effect with radiation in NSCLC $(47,48)$. A preclinical study by Deng et al. showed that PD-L1 expression was upregulated on tumor cells after radiation therapy (47). Studying synergy of RT and PD-1 blockade in the Krasdriven genetically engineered mouse models of NSCLC, Herter-Sprie and Koyama et al. demonstrated that RT leads to an adaptive upregulation of tumor cell PD-L1 expression and that concomitant administration of PD-1 antibody generates effective antitumor immunity and long-term tumor control (49). Furthermore, they demonstrated lack of antineoplastic efficacy of PD-1 blockade in tumors that relapsed after RT, along with significant induction of T cell inhibitory markers in post-RT relapse setting in the murine model, suggesting that most synergy between RT and PD-1 inhibition can be achieved in RT-naïve tumors $(50,51)$.

\section{Role of immunotherapy in combination with RT in treatment of locally advanced NSCLC}

Approximately one quarter of patients with lung cancer are diagnosed with locally advanced NSCLC (LA-NSCLC) (52). Despite definitive multimodality treatment of the primary tumor, the majority of the patients experience disease recurrence (53), with distant failures being the most common patterns of recurrence in adenocarcinoma $(54,55)$. Therefore, innovative strategies have been under investigation aiming to improve long-term disease control in LA-NSCLC patients. One of such novel approaches is harnessing RT's immunomodulatory properties to enhance efficacy of the immune checkpoint inhibitors.

\section{Sequential use of checkpoint inbibitors and $R T$ in locally advanced NSCLC}

Expanding on preclinical work, several clinical trials have 
reported encouraging results in employing combinations of radiation therapy with inhibition of PD-1 signaling pathway as a novel strategy for treatment of LA- NSCLC. Shaverdian et al. (56) conducted a secondary analysis of the expansion NSCLC cohort from the KEYNOTE-001 trial $(57,58)$ aiming to assess efficacy and safety of therapy with the PD-1 inhibitor Pembrolizumab in patients with advanced NSCLC who previously received radiotherapy for treatment of lung cancer. The investigators evaluated ninety-eight patients treated at a single institution who were enrolled on the seminal KEYNOTE-001 trial which was a large international, multicenter, phase 1 trial of single agent pembrolizumab in patients with advanced solid tumors (59). The median PFS was substantially longer in patients who received any type of previous radiation therapy before pembrolizumab (4.4 versus 2.1 months, $\mathrm{HR} 0.56, \mathrm{P}=0.019$ ). Overall survival was also significantly longer in patients with a history of radiation therapy (10.6 versus 5.3 months, HR 0.58, $\mathrm{P}=0.026)$. The safety profile was acceptable, with three patients who had received previous thoracic radiation therapy $v s$. one patient with no previous radiation to the chest developing $\geq$ Grade 3 treatment related pulmonary toxicities (56).

Pembrolizumab was also evaluated as a consolidative therapy in the phase II study conducted by the Hoosier Cancer Research Network LUN 14-179 (60,61). The primary endpoint was time to metastatic disease or death (TMDD).In this trial 93 patients with unresectable stage III A-B NSCLC were treated with pembrolizumab for up to a year following definitive chemoradiation. With a median follow up of 32.2 months, consolidation pembrolizumab improved the TMDD and PFS in comparison to historical controls. The median TMDD was 30.7 months. The median PFS was 18.7 months and the median OS was 35.8 months. The toxicity profile was comparable to that observed in previous pembrolizumab studies. Five patients (5.4\%) experienced Grade 3-4 pneumonitis, and there was one pneumonitis-related death.

The most robust and practice-changing results of investigating immunotherapy for treatment of locally advanced NSCLC in the consolidative setting was reported with PD-L1 inhibition by durvalumab $(7,8)$. The PACIFIC trial made a significant impact on the treatment paradigm for locally advanced NSCLC by becoming the first phase III prospective randomized controlled trial to show an increase in PFS and OS using consolidation durvalumab following concurrent chemoradiation. In this trial, over 700 patients with stage III unresectable NSCLC without disease progression were randomized in a 2:1 ratio to receive up to 12 months of either durvalumab or placebo following concurrent chemoradiation. The initial analysis demonstrated a significantly improved PFS compared to the placebo (16.8 months compared to 5.6 months, $\mathrm{P}<0.001$ ) leading to the FDA and EMA approval of durvalumab for use in the consolidative space of LA-NSCLC patients. Updated analysis showed a significantly improved twoyear overall survival rates in the durvalumab arm $(66.3 \%$ vs. $55.6 \%, \mathrm{P}=0.005)$. The median time to death or distant metastasis was markedly prolonged in durvalumab arm (28.3 vs. 16.2 months, HR 0.53 (95\% Cl: 0.41-0.68). In the 3-year update, median duration of follow up was 33 months. The overall median survival has yet to be reached with durvalumab versus 29.1 months with placebo. The 3 -year OS continued to demonstrate benefit of maintenance durvalumab (57\% vs. 43.5\%). Patient-reported outcomes showed no clinically important differences between the groups suggesting the tolerability of durvalumab $(7,8,62,63)$.

\section{Concurrent use of checkpoint inbibitors and RT in locally advanced NSCLC}

Building upon the success of chemoradiation followed by immunotherapy in treating LA-NSCLC, many trials are evaluating the role of immunotherapy in earlier in the course of therapy. In a completed trial by Jabbour et al., twenty-one patients received pembrolizumab concurrently with chemoradiation therapy (chemoRT) in locally advanced NSCLC (64). Specifically, patients were divided into five cohorts: (I) full dose (FD) pembrolizumab (200 mg intravenously Q3Wk) starting 2-6 weeks after chemoRT; (II) reduced dose (RD) pembrolizumab (100 $\mathrm{mg}$ intravenously Q3Wk) starting Day 29 of chemoRT, (III) FD pembrolizumab starting Day 29 of chemoRT, (IV) RD pembrolizumab starting on Day 1 of chemoRT, (V) FD pembrolizumab starting on Day 1 of chemoRT. All cohorts received Q3Wk of pembrolizumab up to 18 cycles. Dose limiting toxicity (DLT) was defined as grade 4 or higher of pneumonitis within 21 days of the first cycle of pembrolizumab. No DLTs were reported in any cohorts. Grade 3 or higher irAEs were reported in 4 patients. For patients who received greater than two doses, the median PFS was 21 months (95\% CI: 15.3 to infinity). Overall, combined PD-1 treatment and chemoRT was well tolerated with encouraging PFS of $69.7 \%$ at 12 months, however, with potentially an increased risk of pneumonitis using concurrent PD-1 checkpoint blockade (64). 
The phase II DETERRED study aimed to assess the safety and feasibility of concurrent use of atezolizumab with chemoradiotherapy followed by consolidation with atezolizumab in LA-NSCLC $(65,66)$. In this study, immunotherapy was given sequentially in part one $(\mathrm{N}=10)$ or concurrently in part two $(\mathrm{N}=30)$ with chemoradiation, followed by full dose doublet chemotherapy and consolidation atezolizumab. In Part 1, the median PFS was 18.6 months, and the OS was 22.8 months. In Part 2, the median PFS was 13.2 months, and OS was not reached. The PD-L1 status in baseline tumor biopsy was evaluable for 34 patients. There were no significant differences in cancer recurrence based on PD-L1 expression of $<1 \%$ $v s . \geq 1 \%$ ( $56.3 \%$ vs. $38.9 \%$, respectively), or for the $\mathrm{PD}-$ L1 cutoff of less or more than $50 \%(53.8 \%$ vs. $25 \%)$. There was no additive toxicity as a result of concurrent use of Atezolizumab with RT, and frequency and grade of pneumonitis were similar to that seen in previous trials (four patients developed grade 2 and one patient grade 3 pneumonitis).

The NICOLAS-ETOP trial was conducted in Europe and was a phase II trial which evaluated safety of nivolumab with concurrent chemoRT in unresectable LA-NSCLC (67). In this trial, 82 patients received three cycles of platinumbased chemotherapy concurrently or sequentially with $\mathrm{RT}$. In the concurrent chemoRT regimen, radiotherapy was delivered at $66 \mathrm{~Gy}$ in 33 daily fractions, while for the sequential chemoRT regimen, radiotherapy was delivered in 24 daily fractions of $2.75 \mathrm{~Gy}$. The mean lung dose was restricted to $20 \mathrm{~Gy}$. Nivolumab started concurrently with RT. In the interim analysis, no unexpected adverse effects or safety concerns have been reported. For the first 21 patients, no grade $\geq 3$ pneumonitis was observed in 3 months following completion of RT, while one late event occurred 6 months after completion of RT. One -year PFS will be evaluated in an expanded patient cohort.

\section{Use of immunotherapy with RT in Stage IV patients}

Immunogenic properties of radiotherapy, its ability to convert immunologically "cold" tumors into "hot" tumors capable of inducing immune response has generated a new concept of multisite SBRT as an emerging paradigm for treatment of metastatic disease. Luke et al. evaluated the safety of pembrolizumab and multisite SBRT in 79 patients with solid tumors, seven of who had Stage IV NSCLC (68). The patients received SBRT to a dose $30-50$ Gy in 3-5 fractions delivered to $2-4$ metastatic lesions. Pembrolizumab was started within 7 days from completion of SBRT. With a median follow up of 5.5 months, 6 patients experienced DLT without radiation dose changes. The percent change in tumor diameter for irradiated patients was significantly improved $(-21.7 \%)$ for irradiated patients compared to nonirradiated patients $(1.7 \%)$. In the 68 patients with imaging follow-up, the overall objective response rate was $13.2 \%$. Median PFS and OS were 3.1 and 9.6 months, respectively. Expression of interferon- $\gamma$-associated genes from post-SBRT tumor biopsy specimens significantly correlated with nonirradiated tumor response. Overall, the data shows synergy between SBRT and pembrolizumab in inducing out of field responses (68).

\section{Dual checkpoint blockade in combination with radiation}

The Big Ten Cancer Research Consortium LUN 16-081 is a randomized, multi-center, phase II study, evaluating efficacy and safety of combining a CTLA-4 inhibitor with a PD-1 inhibitor as consolidation therapy following definitive chemoRT in patients with unresectable stage IIIA/IIIB NSCLC. An interim safety analysis has been reported (69). There were no unexpected safety signals in the first 20 patients treated on protocol. Four patients experienced $\geq$ Grade 2 pneumonitis. The incidence of $\geq$ Grade 3 irAEs was higher in the combination arm, however these were manageable with the use of established guidelines, and no therapy related deaths were observed. Pneumonitis rates for all aforementioned trials are summarized in Table 1.

\section{SBRT with immunotherapy in Early Stage NSCLC}

There are several ongoing prospective trials which are investigating the use of SBRT with immunotherapy for medically inoperable early-stage non-small cell lung cancer, included in Table 2. Some of the endpoints being evaluated are safety, progression-free survival, overall survival, pneumonitis rates, hematologic toxicities, and adverse events. While combined immunoradiotherapy has brought substantial benefit in locally advanced NSCLC patients, these ongoing trials will help elucidate whether this benefit is seen in early stage patients as well.

\section{Conclusions}

The combination of conventional RT or SBRT with immunotherapy is a promising treatment option for patients with locally advanced non-small lung cancer. 
Table 1 Pneumonitis rates of various trials

\begin{tabular}{|c|c|c|c|c|}
\hline \multirow{2}{*}{ Name of Study } & \multirow{2}{*}{ \# of Patients } & \multicolumn{3}{|c|}{ Pneumonitis Rates } \\
\hline & & G1/G2 & G3 & G5 \\
\hline KEYNOTE 001 & 24 & \multicolumn{3}{|c|}{ Treatment-related Pneumonitis $12.5 \%$} \\
\hline PACIFIC & 475 & $\geq \mathrm{G} 1=33.9 \%$ & $\geq \mathrm{G} 3=3.4 \%$ & G5 $=0.08 \%$ \\
\hline LUN 14-179 & 93 & $\geq G 2=17.2 \%$ & $\geq G 3=6.5 \%$ & $\mathrm{G} 5=1.07 \%$ \\
\hline DETERRED & 40 & $\geq \mathrm{G} 2=12.5 \%$ & G3=2.5\% & G5 $=0 \%$ \\
\hline Jabbour et al. & 23 & $\geq \mathrm{G} 2=26 \%$ & $\geq \mathrm{G} 3=8.6 \%$ & $\mathrm{G} 5=4.34 \%$ \\
\hline NICOLAS & 80 & $\geq \mathrm{G} 1=42.5 \%$ & $\geq \mathrm{G} 3=10 \%$ & $\mathrm{G} 5=0 \%$ \\
\hline LUN 16-081 & 20 & $\geq \mathrm{G} 2=20 \%$ & $\mathrm{G} 3=5 \%$ & $\mathrm{G} 5=0 \%$ \\
\hline
\end{tabular}

Table 2 Ongoing trials of various immunotherapies with SBRT used in NSCLC

\begin{tabular}{|c|c|c|c|c|}
\hline Name of Study & Drug & Phase & Stage & Clinical Trial \# \\
\hline $\begin{array}{l}\text { Atezolizumab and Stereotactic Body Radiation Therapy in Treating } \\
\text { Patients With Non-small Cell Lung Cancer }\end{array}$ & Atezolizumab & I & I & NCT02599454 \\
\hline $\begin{array}{l}\text { SABR-ATAC: A Trial of TGF-beta Inhibition and Stereotactic Ablative } \\
\text { Radiotherapy for Early Stage Non-small Cell Lung Cancer }\end{array}$ & Fresolimumab & $\mathrm{I} / \mathrm{II}$ & I & NCT02581787 \\
\hline SBRT Combined With Avelumab for Management of Early Stage NSCLC & Avelumab & $\mathrm{I} / \mathrm{II}$ & I & NCT03050554 \\
\hline $\begin{array}{l}\text { Astra Zeneca ISABR Study: Randomized Phase I/II Study of Stereotactic } \\
\text { Body Radiotherapy }\end{array}$ & Durvalumab & $1 / I I$ & $1 / I I$ & NCT03148327 \\
\hline $\begin{array}{l}\text { SBRT With Immunotherapy in Early Stage Non-small Cell Lung Cancer: } \\
\text { Tolerability and Lung Effects (STILE) }\end{array}$ & Nivolumab & $\mathrm{I} / \mathrm{II}$ & $\mathrm{I} / \mathrm{II}$ & NCT03383302 \\
\hline Ablative STEreotactic RadiOtherapy wlth Durvalumab (ASTEROID) & Durvalumab & II & I & NCT03446547 \\
\hline $\begin{array}{l}\text { Stereotactic Body Radiation Therapy With or Without Nivolumab in } \\
\text { Treating Patients With Stage I-IIA or Recurrent NSCLC }\end{array}$ & Nivolumab & II & $1 / I I$ & NCT03110978 \\
\hline Durvalumab With or Without SBRT in Clinical Stage I, II and IIIA NSCLC & Durvalumab & II & I/II/III & NCT02904954 \\
\hline $\begin{array}{l}\text { Durvalumab and Consolidation SBRT Following CRT for Locally Advanced } \\
\text { Stage III NSCLC }\end{array}$ & Durvalumab & II & III & NCT03589547 \\
\hline $\begin{array}{l}\text { FLT3 Ligand Immunotherapy and Stereotactic Radiotherapy for Advanced } \\
\text { Non-small Cell Lung Cancer (FLT3) }\end{array}$ & $\begin{array}{l}\text { FLT3 Ligand Therapy } \\
\text { (CDX-301) }\end{array}$ & II & III/IV & NCT02839265 \\
\hline $\begin{array}{l}\text { Durvalumab vs. Placebo Following Stereotactic Body Radiation Therapy in } \\
\text { Early Stage Unresected Non-small Cell Lung Cancer Patients (PACIFIC-4) }\end{array}$ & Durvalumab & III & $\mathrm{I} / \mathrm{II}$ & NCT03833154 \\
\hline $\begin{array}{l}\text { Efficacy and Safety Study of Stereotactic Body Radiotherapy (SBRT) With } \\
\text { or Without Pembrolizumab in Adults With Medically Inoperable Stage I or } \\
\text { IIA Non-Small Cell Lung Cancer (Keynote-867) }\end{array}$ & Pembrolizumab & III & $\mathrm{I} / \mathrm{II}$ & NCT03924869 \\
\hline $\begin{array}{l}\text { Testing the Addition of the Drug Atezolizumab to the Usual Radiation } \\
\text { Treatment for Patients With Early Non-small Cell Lung Cancer }\end{array}$ & Atezolizumab & III & I/II & NCT04214262 \\
\hline
\end{tabular}


Further research will be required on mitigating toxicities in these patients. Ongoing trials will delineate subsets of patients that can derive meaningful benefit by the addition of immunotherapy to radiation therapy, while helping to identify the patients at risk for heightened toxicity from the addition of RT to immunotherapy, thereby further improving the survival outcomes of patients with lung cancer.

\section{Acknowledgments}

Funding: Grant: P30CA072720.

\section{Footnote}

Reporting Checklist: The authors have completed the Narrative Review reporting checklist. Available at: http:// dx.doi.org/10.21037/shc-20-66

Conflicts of Interest: All authors have completed the ICMJE uniform disclosure form (available at http:// dx.doi.org/10.21037/shc-20-66). JM reports personal fees from AstraZeneca, outside the submitted work; MP reports personal fees from Bayer Corporation, outside the submitted work; SJ reports grants, personal fees and nonfinancial support from Merck, outside the submitted work. The other authors have no conflicts of interest to declare.

Ethical Statement: The authors are accountable for all aspects of the work in ensuring that questions related to the accuracy or integrity of any part of the work are appropriately investigated and resolved.

Open Access Statement: This is an Open Access article distributed in accordance with the Creative Commons Attribution-NonCommercial-NoDerivs 4.0 International License (CC BY-NC-ND 4.0), which permits the noncommercial replication and distribution of the article with the strict proviso that no changes or edits are made and the original work is properly cited (including links to both the formal publication through the relevant DOI and the license). See: https://creativecommons.org/licenses/by-nc-nd/4.0/.

\section{References}

1. Bray F, Ferlay J, Soerjomataram I, et al. Global cancer statistics 2018: GLOBOCAN estimates of incidence and mortality worldwide for 36 cancers in 185 countries. CA Cancer J Clin 2018;68:394-424.
2. Roviaro G, Varoli F, Vergani C, et al. Long-term survival after videothoracoscopic lobectomy for stage I lung cancer. Chest 2004;126:725-32.

3. Cao J, Yuan P, Wang Y, et al. Survival Rates After Lobectomy, Segmentectomy, and Wedge Resection for Non-Small Cell Lung Cancer. Ann Thorac Surg 2018;105:1483-91.

4. Chang JY, Senan S, Paul MA, et al. Stereotactic ablative radiotherapy versus lobectomy for operable stage I nonsmall-cell lung cancer: a pooled analysis of two randomised trials. Lancet Oncol 2015;16:630-7.

5. Timmerman R. Stereotactic Body Radiation Therapy for Inoperable Early Stage Lung Cancer. JAMA 2010;303:1070.

6. Timmerman RD, Hu C, Michalski J, et al. Long-term Results of RTOG 0236: A Phase II Trial of Stereotactic Body Radiation Therapy (SBRT) in the Treatment of Patients with Medically Inoperable Stage I NonSmall Cell Lung Cancer. Int J Radiat Oncol Biol Phys 2014;90:S30.

7. Antonia SJ, Villegas A, Daniel D, et al. Durvalumab after Chemoradiotherapy in Stage III Non-Small-Cell Lung Cancer. N Engl J Med 2017;377:1919-29.

8. Antonia SJ, Villegas A, Daniel D, et al. Overall Survival with Durvalumab after Chemoradiotherapy in Stage III NSCLC. N Engl J Med 2018;379:2342-50.

9. Hu ZI, Ho AY, McArthur HL. Combined Radiation Therapy and Immune Checkpoint Blockade Therapy for Breast Cancer. Int J Radiat Oncol Biol Phys 2017;99:153-64.

10. Eriksson D, Stigbrand T. Radiation-induced cell death mechanisms. Tumour Biol 2010;31:363-72.

11. Gupta A, Probst HC, Vuong V, et al. Radiotherapy Promotes Tumor-Specific Effector CD8+ T Cells via Dendritic Cell Activation. J Immunol 2012;189:558-66.

12. Sharabi AB, Nirschl CJ, Kochel CM, et al. Stereotactic Radiation Therapy Augments Antigen-Specific PD1-Mediated Antitumor Immune Responses via CrossPresentation of Tumor Antigen. Cancer Immunol Res 2015;3:345-55.

13. Burdette DL, Vance RE. STING and the innate immune response to nucleic acids in the cytosol. Nat Immunol 2013;14:19-26.

14. Deng L, Liang H, Xu M, et al. STING-Dependent Cytosolic DNA Sensing Promotes Radiation-Induced Type I Interferon-Dependent Antitumor Immunity in Immunogenic Tumors. Immunity 2014;41:843-52.

15. Woo SR, Fuertes MB, Corrales L, et al. STING- 
dependent cytosolic DNA sensing mediates innate immune recognition of immunogenic tumors. Immunity 2014;41:830-42.

16. Burnette BC, Liang H, Lee Y, et al. The Efficacy of Radiotherapy Relies upon Induction of Type I InterferonDependent Innate and Adaptive Immunity. Cancer Res 2011;71:2488-96.

17. Lugade AA, Moran JP, Gerber SA, et al. Local Radiation Therapy of B16 Melanoma Tumors Increases the Generation of Tumor Antigen-Specific Effector Cells That Traffic to the Tumor. J Immunol 2005;174:7516-23.

18. Hong CSCCYTPJH. Rapid induction of cytokine gene expression in the lung after single and fractionated doses of radiation. Int J Radiat Biol 1999;75:1421-7.

19. Hallahan DE, Spriggs DR, Beckett MA, et al. Increased tumor necrosis factor alpha mRNA after cellular exposure to ionizing radiation. Proc Natl Acad Sci U S A 1989;86:10104-7.

20. Rodriguez-Ruiz ME, Garasa S, Rodriguez I, et al. Intercellular Adhesion Molecule-1 and Vascular Cell Adhesion Molecule Are Induced by Ionizing Radiation on Lymphatic Endothelium. Int J Radiat Oncol Biol Phys 2017;97:389-400.

21. Matsumura S, Demaria S. Up-regulation of the Proinflammatory Chemokine CXCL16 is a Common Response of Tumor Cells to Ionizing Radiation. Radiat Res 2010;173:418-25.

22. Matsumura S, Wang B, Kawashima N, et al. RadiationInduced CXCL16 Release by Breast Cancer Cells Attracts Effector T Cells. J Immunol 2008;181:3099-107.

23. Mole RH. Whole body irradiation; radiobiology or medicine? Br J Radiol 1953;26:234-41.

24. Chakraborty M, Abrams SI, Camphausen K, et al. Irradiation of Tumor Cells Up-Regulates Fas and Enhances CTL Lytic Activity and CTL Adoptive Immunotherapy. J Immunol 2003;170:6338-47.

25. Kuwabara $M$, Takahashi K, Inanami O. Induction of Apoptosis through the Activation of SAPK/JNK Followed by the Expression of Death Receptor Fas in X-irradiated Cells. J Radiat Res 2003;44:203-9.

26. Motz GT, Santoro SP, Wang L-P, et al. Tumor endothelium FasL establishes a selective immune barrier promoting tolerance in tumors. Nat Med 2014;20:607-15.

27. Yang J-Y, Xia W, Hu M. Ionizing radiation activates expression of FOXO3a, Fas ligand, and Bim, and induces cell apoptosis. Int J Oncol 2006;29:643-8.

28. Chinnaiyan AM, Prasad U, Shankar S, et al. Combined effect of tumor necrosis factor-related apoptosis-inducing ligand and ionizing radiation in breast cancer therapy. Proc Natl Acad Sci U S A 2000;97:1754-9.

29. Marini P, Schmid A, Jendrossek V, et al. Irradiation specifically sensitises solid tumour cell lines to TRAIL mediated apoptosis. BMC Cancer 2005;5:5.

30. Kim J-Y, Son Y-O, Park S-W, et al. Increase of NKG2D ligands and sensitivity to NK cell-mediated cytotoxicity of tumor cells by heat shock and ionizing radiation. Exp Mol Med 2006;38:474-84.

31. Rosen EM, Fan S, Rockwell S, et al. The molecular and cellular basis of radiosensitivity: implications for understanding how normal tissues and tumors respond to therapeutic radiation. Cancer Invest 1999;17:56-72.

32. Yovino S, Kleinberg L, Grossman SA, et al. The Etiology of Treatment-related Lymphopenia in Patients with Malignant Gliomas: Modeling Radiation Dose to Circulating Lymphocytes Explains Clinical Observations and Suggests Methods of Modifying the Impact of Radiation on Immune Cells. Cancer Invest 2013;31:140-4.

33. Darragh LB, Oweida AJ, Karam SD. Overcoming Resistance to Combination Radiation-Immunotherapy: A Focus on Contributing Pathways Within the Tumor Microenvironment. Front Immunol 2019;9:3154.

34. Schaue D, Ratikan JA, Iwamoto KS, et al. Maximizing tumor immunity with fractionated radiation. Int J Radiat Oncol Biol Phys 2012;83:1306-10.

35. Vanpouille-Box C, Alard A, Aryankalayil MJ, et al. DNA exonuclease Trex1 regulates radiotherapy-induced tumour immunogenicity. Nat Commun 2017;8:15618.

36. Hanahan D, Weinberg RA. Hallmarks of cancer: the next generation. Cell 2011;144:646-74.

37. Ribas A, Wolchok JD. Cancer immunotherapy using checkpoint blockade. Science 2018;359:1350-5.

38. Murillo O, Arina A, Hervas-Stubbs S, et al. Therapeutic antitumor efficacy of anti-CD137 agonistic monoclonal antibody in mouse models of myeloma. Clin Cancer Res 2008;14:6895-906.

39. Chen H, Liakou CI, Kamat A, et al. Anti-CTLA-4 therapy results in higher CD4+ICOShiT cell frequency and IFN- $\gamma$ levels in both nonmalignant and malignant prostate tissues. Proc Natl Acad Sci U S A 2009;106:2729-34.

40. Ji R-R, Chasalow SD, Wang L, et al. An immuneactive tumor microenvironment favors clinical response to ipilimumab. Cancer Immunol Immunother 2012;61:1019-31.

41. Tang C, Welsh JW, De Groot P, et al. Ipilimumab with Stereotactic Ablative Radiation Therapy: Phase I Results 
and Immunologic Correlates from Peripheral T Cells. Clin Cancer Res 2017;23:1388-96.

42. Dewan MZ, Galloway AE, Kawashima N, et al. Fractionated but not single-dose radiotherapy induces an immune-mediated abscopal effect when combined with anti-CTLA-4 antibody. Clin Cancer Res 2009; 15:5379-88.

43. Demaria S, Kawashima N, Yang AM, et al. Immunemediated inhibition of metastases after treatment with local radiation and CTLA-4 blockade in a mouse model of breast cancer. Clin Cancer Res 2005;11:728-34.

44. Formenti SC, Rudqvist N-P, Golden E, et al. Radiotherapy induces responses of lung cancer to CTLA-4 blockade. Nat Med 2018;24:1845-51.

45. Sharpe AH, Pauken KE. The diverse functions of the PD1 inhibitory pathway. Nat Rev Immunol 2018;18:153-67.

46. Wherry EJ. T cell exhaustion. Nat Immunol 2011;12:492-9.

47. Deng L, Liang H, Burnette B, et al. Irradiation and antiPD-L1 treatment synergistically promote antitumor immunity in mice. J Clin Invest 2014;124:687-95.

48. Dovedi SJ, Adlard AL, Lipowska-Bhalla G, et al. Acquired Resistance to Fractionated Radiotherapy Can Be Overcome by Concurrent PD-L1 Blockade. Cancer Res 2014;74:5458-68.

49. Herter-Sprie GS, Koyama S, Korideck H, et al. Synergy of radiotherapy and PD-1 blockade in Kras-mutant lung cancer. JCI Insight 2016;1:e87415.

50. Spranger S, Spaapen RM, Zha Y, et al. Up-Regulation of PD-L1, IDO, and Tregs in the Melanoma Tumor Microenvironment Is Driven by CD8+ T Cells. Sci Transl Med 2013;5:200ra116-200ra1.

51. Twyman-Saint Victor C, Rech AJ, Maity A, et al. Radiation and dual checkpoint blockade activate nonredundant immune mechanisms in cancer. Nature 2015;520:373-7.

52. Lee CB, Stinchcombe TE, Rosenman JG, et al. Therapeutic advances in local-regional therapy for stage III non-small-cell lung cancer: evolving role of doseescalated conformal (3-dimensional) radiation therapy. Clin Lung Cancer 2006;8:195-202.

53. Senan S, Brade A, Wang LH, et al. PROCLAIM: Randomized Phase III Trial of Pemetrexed-Cisplatin or Etoposide-Cisplatin Plus Thoracic Radiation Therapy Followed by Consolidation Chemotherapy in Locally Advanced Nonsquamous Non-Small-Cell Lung Cancer. J Clin Oncol 2016;34:953-62.

54. Garg S, Gielda BT, Kiel K, et al. Patterns of locoregional failure in stage III non-small cell lung cancer treated with definitive chemoradiation therapy. Pract Radiat Oncol 2014;4:342-8.

55. Nygard L, Vogelius IR, Fischer BM, et al. A Competing Risk Model of First Failure Site after Definitive Chemoradiation Therapy for Locally Advanced NonSmall Cell Lung Cancer. J Thorac Oncol 2018;13:559-67.

56. Shaverdian N, Lisberg AE, Bornazyan K, et al. Previous radiotherapy and the clinical activity and toxicity of pembrolizumab in the treatment of non-small-cell lung cancer: a secondary analysis of the KEYNOTE-001 phase 1 trial. Lancet Oncol 2017;18:895-903.

57. Garon EB, Hellmann MD, Rizvi NA, et al. Five-Year Overall Survival for Patients With Advanced NonSmallCell Lung Cancer Treated With Pembrolizumab: Results From the Phase I KEYNOTE-001 Study. J Clin Oncol 2019;37:2518-27.

58. Garon EB, Rizvi NA, Hui R, et al. Pembrolizumab for the treatment of non-small-cell lung cancer. N Engl J Med 2015;372:2018-28.

59. Patnaik A, Kang SP, Rasco D, et al. Phase I Study of Pembrolizumab (MK-3475; Anti-PD-1 Monoclonal Antibody) in Patients with Advanced Solid Tumors. Clin Cancer Res 2015;21:4286-93.

60. Durm GA, Althouse SK, Sadiq AA, et al. Phase II trial of concurrent chemoradiation with consolidation pembrolizumab in patients with unresectable stage III nonsmall cell lung cancer: Hoosier Cancer Research Network LUN 14-179. J Clin Oncol 2018;36:8500.

61. Durm GA, Jabbour SK, Althouse SK, et al. A phase 2 trial of consolidation pembrolizumab following concurrent chemoradiation for patients with unresectable stage III non-small cell lung cancer: Hoosier cancer research network LUN 14-179. Cancer 2020;126:4353-61.

62. Hui R, Özgüroğlu M, Villegas A, et al. Patient-reported outcomes with durvalumab after chemoradiotherapy in stage III, unresectable non-small-cell lung cancer (PACIFIC): a randomised, controlled, phase 3 study. Lancet Oncol 2019;20:1670-80.

63. Gray JE, Villegas A, Daniel D, et al. Brief report: Three-year overall survival with durvalumab after chemoradiotherapy in Stage III NSCLC - Update from PACIFIC. J Thorac Oncol 2020;15:288-93.

64. Jabbour SK, Berman AT, Decker RH, et al. Phase 1 Trial of Pembrolizumab Administered Concurrently With Chemoradiotherapy for Locally Advanced Non-Small Cell Lung Cancer: A Nonrandomized Controlled Trial. JAMA Oncol 2020;6:848-55. 
65. Lin SH, Lin Y, Price J, et al. DETERRED: PD-L1 blockade to evaluate the safety of lung cancer therapy using carboplatin, paclitaxel, and radiation combined with MPDL3280A (atezolizumab). J Clin Oncol 2017;35:3064.

66. Lin SH, Lin Y, Yao L, et al. Phase II Trial of Concurrent Atezolizumab With Chemoradiation for Unresectable NSCLC. J Thorac Oncol. 2020;15(2):248-257

67. Peters S, Felip E, Dafni U, et al. Safety evaluation of nivolumab added concurrently to radiotherapy in a standard first line chemo-radiotherapy regimen in stage III non-small cell lung cancer-The ETOP NICOLAS trial.

doi: $10.21037 /$ shc-20-66

Cite this article as: Modi C, Berim L, Isserow L, Malhotra J, Patel M, Langenfeld J, Aisner J, Almeldin D, Jabbour S. Combining radiation therapy and immunotherapy for lung cancers: a narrative review. Shanghai Chest 2021;5:10.
Lung Cancer 2019;133:83-7.

68. Luke JJ, Lemons JM, Karrison TG, et al. Safety and Clinical Activity of Pembrolizumab and Multisite Stereotactic Body Radiotherapy in Patients With Advanced Solid Tumors. J Clin Oncol 2018;36:1611-8.

69. Yan M, Durm GA, Mamdani H, et al. Interim safety analysis of consolidation nivolumab and ipilimumab versus nivolumab alone following concurrent chemoradiation for unresectable stage IIIA/IIIB NSCLC: Big Ten Cancer Research Consortium LUN 16-081. J Clin Oncol 2019;37:8535 